U.S. Department of the Interior

U.S. Geological Survey

\title{
Descriptions of Selected Digital Spatial Data for Ravenna Army Ammunition Plant, Ohio
}

By Charles W. Schalk and Robert A. Darner

Open-File Report 97-802

Prepared in cooperation with the

U.S. Army Industrial Operations Command

Columbus, Ohio

January 1998

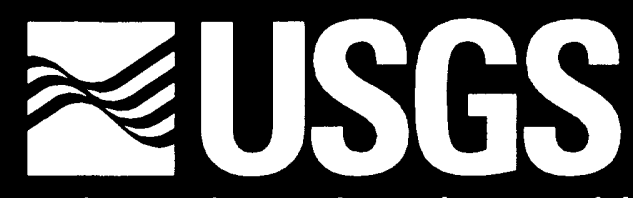

science for a changing world 


\section{U.S. DEPARTMENT OF THE INTERIOR}

BRUCE BABBITT, Secretary

\section{U.S. GEOLOGICAL SURVEY}

Mark Schaefer, Acting Director

Any use of trade, product, or firm names is for descriptive purposes only and does not constitute endorsement by the U.S. Government.

For additional information write to:

District Chief

U.S. Geological Survey

975 West Third Avenue

Columbus, Ohio $43212-3192$
Copies of this report can be purchased from:

U.S. Geological Survey

Information Services

Box 25286

Denver Federal Center

Denver, CO 80225-0286 


\section{CONTENTS}

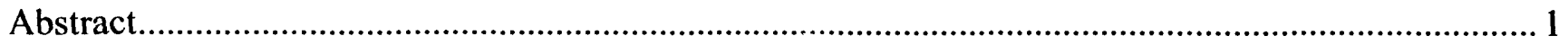

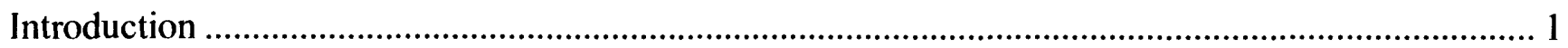

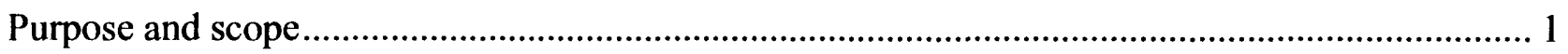

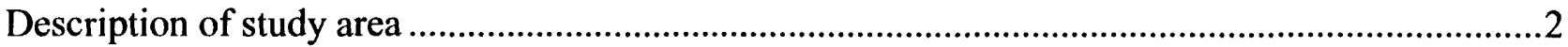

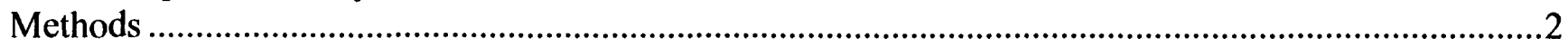

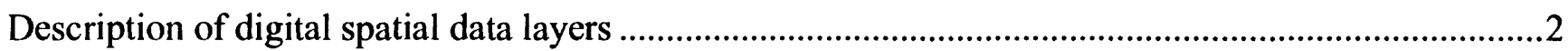

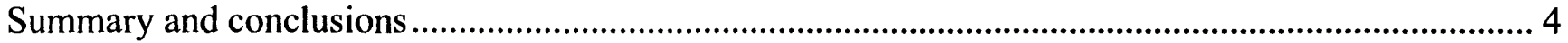

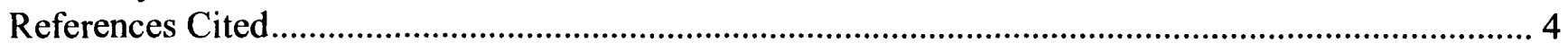

FIGURE

1. Map showing location of Ravenna Army Ammunition Plant, Ohio ...................................... 3

\section{TABLE}

1. Data layers included in the digital spatial data base for Industrial Operations Command.

\section{CONVERSION FACTORS AND VERTICAL DATUM}

\begin{tabular}{rccl}
\hline Multiply & By & To obtain \\
\hline & Length & \\
\hline inch (in.) & 2.54 & centimeter \\
inch (in.) & 25.4 & millimeter \\
& foot (ft) & 0.3048 & meter \\
mile (mi) & 1.609 & kilometer \\
& & Area & \\
\hline & acre & 4.047 & square meter \\
& acre & 0.4047 & hectare \\
& &
\end{tabular}

Temperature in degrees Fahrenheit $\left({ }^{\circ} \mathrm{F}\right)$ may be converted to degrees Celsius $\left({ }^{\circ} \mathrm{C}\right)$ as follows:

$$
{ }^{\circ} \mathrm{C}=\left({ }^{\circ} \mathrm{F}-32\right) / 1.8
$$

Sea level: In this report, "sea level" refers to the National Geodetic Vertical Datum of 1929 (NGVD of 1929) - a geodetic datun derived from a general adjustment of the first-order level nets of both the United States and Canada, formerly called Sea Level Datum of 1929. 


\title{
Descriptions of Selected Digital Spatial Data for Ravenna Army Ammunition Plant, Ohio
}

\author{
B y Charles W. Schalk and Robert A. Darner
}

\section{ABSTRACT}

Digital spatial data of Ravenna Army Ammunition Plant (RVAAP), in northeastern Ohio, were compiled or generated from existing maps for U.S. Army Industrial Operations Command. The data are in the Ohio north state-plane coordinate system (North American Datum of 1983 ) in an ARC/INFO geographic information system format. The data comprise 15 layers, which include boundaries, topography, and natural and cultural features. An additional layer comprises scanned and rectified aerial photographs of RVAAP.

\section{INTRODUCTION}

U.S. Army activities on Ravenna Army Ammunition Plant (RVAAP), in Trumbull and Portage Counties, Ohio, have scaled back considerably since the 1940 's. Facilities on the 21,000 -acre ammunition plant included 1,700 buildings (of which 750 were for munitions storage), $210 \mathrm{mi}$ of road, $110 \mathrm{mi}$ of railroad, and numerous sewage, water-treatment, and steam plants. The plant was used actively during World War II, the Korean War, and the Vietnam War. Primary activities on RVAAP included load assembling and packing and storage of ammunition and explosives. Some conventional weapons systems were tested and developed on the facility, and obsolete weapons were decommissioned. Since 1992, RVAAP has been in the process of realignment and closure because it is no longer needed as a production facility.

Army Industrial Operations Command (IOC) is the primary facility host. Currently, the mission of RVAAP is the storage of munitions and the restoration and management of the environment. Other groups, including the Ohio Army National Guard (OHARNG) and Air Force Reserves, use parts of the facility for troop training. Normal operations on RVAAP are overseen by about 35 people, most of whom are employees of a security contractor. As part of its mission to restore and manage the environment, the U.S. Army is involved in and committed to the cleanup of potential contamination at 38 areas of concern on RVAAP. Because geographic information systems (GIS) technology has been shown to be an efficient means of compiling, storing, retrieving, and using spatial data for remedial activities, the IOC contracted with the U.S. Geological Survey (USGS) to build a data base of digital spatial data for IOC use.

Some digital spatial data were already available as a result of a project by OHARNG and the USGS, which was completed in early 1997 . These data layers were used as the foundation of the work described in this report.

\section{Purpose and Scope}

This report describes the digital spatial data base of (RVAAP) created for IOC. The data were created and processed in ARC/INFO GIS format. Some of the data layers that were created for OHARNG were expanded and (or) otherwise revised for IOC to encompass all of RVAAP. Other data layers were not revised. One data layer was created for this project alone. The Army can use these data for remedial and environmental programs on RVAAP. 


\section{Description of Study Area}

RVAAP includes parts of Portage and Trumbull Counties in northeastern Ohio (fig. 1). The cities of Ravenna, Windham, and Newton Falls are within $3 \mathrm{mi}$ of its boundary. It is in the drainage basin of West Branch Mahoning River. The major streams on RVAAP are Hinckley Creek, which flows southward through the western part of RVAAP; Sand Creek, which flows eastward through the center of RVAAP; and South Fork Eagle Creek, which flows eastward along the north boundary of RVAAP to its confluence with Sand Creek. These three streams drain about 14,700 acres, or 69 percent of RVAAP.

Most of the ammunition plant is reverting back to a natural state. Landscaping maintenance is done only along fence lines and around storage bunkers. Roads and railroads are used infrequently and almost exclusively for munitions-storage activities. Woodlands and marshes are common in all areas of RVAAP except OHARNG training grounds $\mathrm{A}, \mathrm{B}$, and $\mathrm{C}$ in the eastern part of RVAAP (fig. 1).

RVAAP is in the glaciated Allegheny Plateau of the Appalachian Plateaus Physiographic Province (Fenneman and Johnson, 1946). The terrain is gently rolling. Topographic relief is about $290 \mathrm{ft}$; the high point is about $1,220 \mathrm{ft}$ above sea level in the northwest corner, and the low point is about $930 \mathrm{ft}$ above sea level in the southeast corner. Soils on the ammunition plant are mostly silt loams. Some loams, loamy sands, and mucks can be found in the bottoms of South Fork Eagle Creek. Other loams and loamy sands can be found in a few parts of Area $\mathrm{C}$ and in the bottoms of Sand Creek. The soil series most common to RVAAP is the Mahoning-Ellsworth association, which consists of nearly level to sloping, somewhat poorly drained to moderately well drained soils that formed mostly in moderately fine textured glacial till on uplands. Some of the soils on the eastern part of RVAAP are in the SebringHolly-Caneadea association, which are mostly level, poorly drained soils formed in lacustrine material. Details concerning the soils on RVAAP can be found in Ritchie and others (1978) and Williams (1992).

About 37 in. of precipitation per year falls on RVAAP, including an average of 54 in. of snow (National Oceanic and Atmospheric Administration, 1991). Average temperature ranges from $24.8^{\circ} \mathrm{F}$ in January to $71.9^{\circ} \mathrm{F}$ in July. The average growing season is 165 days, from about April 28 to October 18.

\section{METHODS}

Most of the GIS data layers were digitized from 1:24,000-scale topographic quadrangle maps (Ravenna, Windham, and Newton Falls quadrangles). Some were obtained from agencies other than the USGS, and one was created from files downloaded from on-line sources. The BUILDING data layer was digitized from aerial-photograph images, which are described in the next paragraph. A compilation of metadata (data about the data) of these data layers is presented at the back of this report. All data layers were peer reviewed for accuracy in linework, annotation, and documentation.

High-altitude aerial photographs $(1: 40,000$ scale) of RVAAP were obtained from the USGS EROS Data Center in black-and-white formats ${ }^{\top}$. Digital images of the three black-and-white aerial photographs were created by scanning the 9 -in. by 9 -in. film positives at a density of $10 \mu \mathrm{m}$ (2,540 dots per in.). The images were saved as tag image file format (TIFF) files and were rectified into state-plane coordinates by use of ARC/INFO image-processing software.

\section{DESCRIPTION OF DIGITAL SPATIAL DATA LAYERS}

The data layers described in this report and delivered to IOC are listed in table 1. More detailed descriptions of each data layer (except the aerial photography images) begin on page 6 . Documentation of these data and descriptions are also included in the digital data layers (INFO *.nar files) delivered to IOC.

\footnotetext{
'Aerial photographs were part of the National Aerial Photography Program (NAPP), image scale 1:40,000. Black-and-white photographs included Cleveland South frames 7930-40, 7930-82, and 7934-2 14 (April 1994).
} 


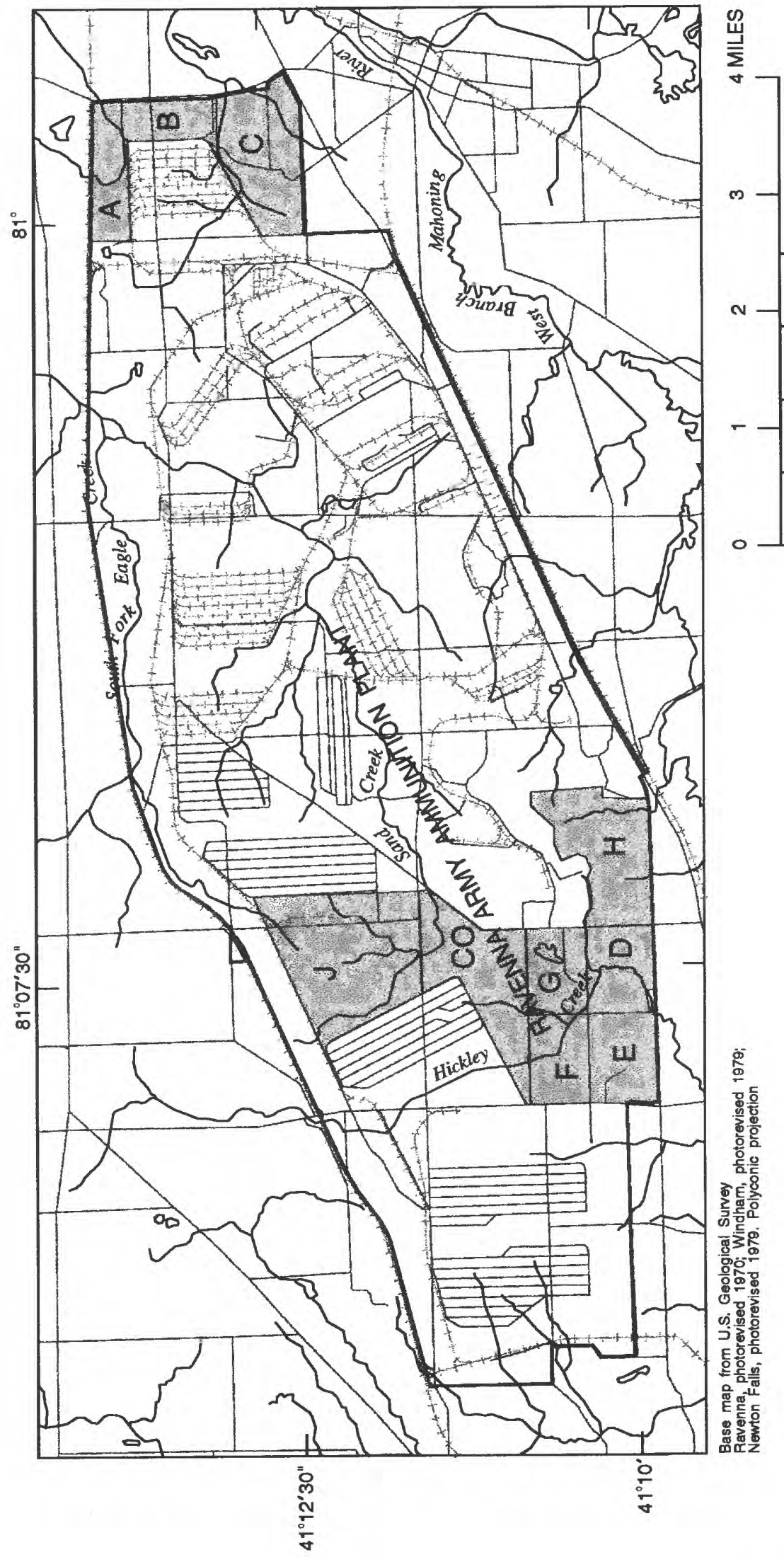

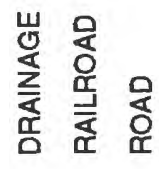

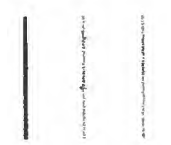

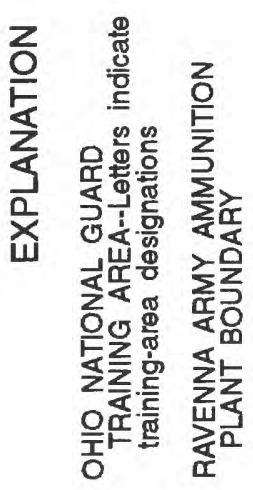

응

름

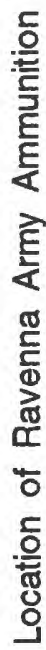

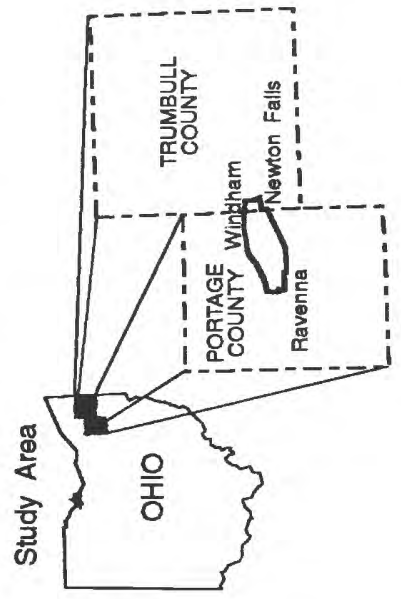

을 
Table 1. Data layers included in the digital spatial data base for Industrial Operations Command [Three-quadrangle extent spans Ravenna, Windham, and Newton Falls 1:24,000-scale topographic quadrangles; plant extent includes the ammunition plant and a 0.5 -mile buffer zone around its boundary]

\begin{tabular}{|c|c|c|}
\hline Data layer & Extent & Source \\
\hline County boundaries & Three quadrangles & OEPA $^{\top}$ \\
\hline Ammunition-plant boundary & Plant & Quads $^{2}$ \\
\hline Municipal boundaries & Three quadrangles & Quads $^{2}$ \\
\hline Township boundaries & Three quadrangles & OEPA $^{\prime}$ \\
\hline Roads & Three quadrangles & Quads $^{2}$ \\
\hline Railroads & Three quadrangles & Quads $^{2}$ \\
\hline Pipelines (off-plant) & Three quadrangles & Quads $^{2}$ \\
\hline Surface drainage & Three quadrangles & Quads $^{2}$ \\
\hline Topography & Three quadrangles & $w w w^{3}$ \\
\hline Soils & Plant & USDA $^{4}$ \\
\hline Plant communities & Plant & ODNR $^{5}$ \\
\hline Woodlands & Plant & Quads $^{2}$ \\
\hline National Wetland Inventory areas & Plant & $\mathrm{FWS}^{6}$ \\
\hline Buildings & Plant & NAPP $^{7}$ \\
\hline Training areas & Plant & OHARNG $^{8}$ \\
\hline Georeferenced aerial photographs (TIFF) & Plant & $\mathrm{NAPP}^{7}$ \\
\hline \multicolumn{3}{|c|}{$\begin{array}{l}\text { 1 Part of a statewide township layer provided by Dave Wilburn, Ohio Environmental Protection Agency, July 1997, scale 1:24,00 } \\
2 \text { Digitized from Ravenna (photoinspected 1977). Windham (photorevised 1979), and Newton Falls (photorevised 1979), Ohio, } \\
\text { 1:24,000-scale U.S. Geological Survey topographic quadrangles. } \\
{ }^{3} \text { Obtained from Ohio Department of Administrative Services data repository on the World Wide Web, scale 1:24,000, } \\
\text { http://www.geodata.state.oh.us/dlg/, June 1997. } \\
{ }^{4} \text { Digitized from Portage and Trumbull County soils maps by the U.S. Department of Agriculture, Ritchie and others (1978), and } \\
\text { Williams (1992), scale 1:15,840. } \\
{ }^{5} \text { Digitized from a map provided by Ohio Department of Natural Resources (Andreas, 1993), scale approximately 1:24,000. } \\
{ }^{6} \text { Digitized from U.S. Fish and Wildlife Service National Wetland Inventory maps (1977a-c), scale I:24,000. } \\
{ }^{7} \text { Scanned from photographs that were obtained as part of the National Aerial Photography Program, U.S. Geological Survey. } \\
{ }^{8} \text { Boundaries digitized from verbal descriptions provided by Ohio Army National Guard. }\end{array}$} \\
\hline
\end{tabular}

\section{SUMMARY AND CONCLUSIONS}

Sixteen geographic information system (GIS) data layers, including georeferenced aerial photographs, were compiled or generated from numerous sources for U.S. Army Industrial Operations Command (IOC). All of the data layers cover Ravenna Army Ammunition Plant (RVAAP) in northeastern Ohio. Most of the data layers were digitized from 1:24,000scale USGS topographic quadrangle maps (Ravenna, Windhain, and Newton Falls quadrangles), but some were digitized or otherwise obtained from other agencies.

These data can be useful for remediation or construction projects on RVAAP. The scale of most of these data layers is $1: 24,000$, which is suitable for wide-area analysis.
Except for the aerial photographs, which are in tag image file format (TIFF), the data were created and delivered to IOC in ARC/INFO format. Documentation of the data is included on-line in INFO *.nar files. Use of the data in any GIS other than ARC/INFO requires conversion of the data.

\section{REFERENCES CITED}

Andreas, B.K., 1993, Plant communities, in Ohio Department of Natural Resources, 1993, Species and plant communities inventory, Ravenna Army Ammunition Plant: Report to Ravenna Army Ammunition Plant [variously paginated]. 
Fenneman, N.M., and Johnson, D.W., 1946, Physical divisions of the United States: U.S. Geological Survey, scale $1: 7,000,000$.

National Oceanic and Atmospheric Administration, 1991, Monthly station normals of temperature, precipitation, and heating and cooling degree days, 1961-1990, Ohio: Climatography of the United States, no. 81 [not paginated].

Ritchie, A., Bauder, J.R., and Christman, R.L., 1978, Soil survey of Portage County, Ohio: U.S. Department of Agriculture, Soil Conservation Service, 116 p. with maps.
U.S. Fish and Wildlife Service, U.S. Department of the Interior, 1977a, National Wetlands Inventory, Newton Falls, Ohio: Scale 1:24,000.

1977b, National Wetlands Inventory, Ravenna, Ohio: Scale 1:24,000.

$-1977 \mathrm{c}$, National Wetlands Inventory, Windham, Ohio: Scale 1:24,000.

Williams, N.L., 1992, Soil survey of Trumbull County, Ohio: U.S. Department of Agriculture, Soil Conservation Service, 256 p. with maps. 
County boundaries - data layer COUNTY

Description of DOUBLE precision data layer COUNTY

\begin{tabular}{|c|c|c|}
\hline FEATURE CLASSES & $\begin{array}{l}\text { ARCS } \\
\text { POLYGONS } \\
\text { NODES } \\
\text { ANNOTATIONS }\end{array}$ & $\begin{array}{l}3 \\
3 \text { (TOPOLOGY) } \\
2 \\
1656\end{array}$ \\
\hline SECONDARY FEATURES & $\begin{array}{l}\text { Tics } \\
\text { Arc Segments } \\
\text { Polygon Labels }\end{array}$ & $\begin{array}{l}53 \\
191 \\
2\end{array}$ \\
\hline TOLERANCES & $\begin{array}{l}\text { Fuzzy }= \\
\text { Dangle = }\end{array}$ & $\begin{array}{l}328.155 \mathrm{~V} \\
328.155 \mathrm{~N}\end{array}$ \\
\hline \multicolumn{3}{|l|}{ DATA LAYER BOUNDARY } \\
\hline $\begin{array}{ll}\mathrm{Xmin}= & 2272948.500 \\
\mathrm{Ymin}= & 483601.781\end{array}$ & $\begin{array}{l}X \max = \\
Y \max =\end{array}$ & $\begin{array}{l}2514171.250 \\
674502.000\end{array}$ \\
\hline \multicolumn{3}{|c|}{ COORDINATE SYSTEM DESCRIPTION } \\
\hline $\begin{array}{ll}\text { Projection } & \text { STATEPLANE } \\
\text { Datum } & \text { NAD83 } \\
\text { Spheroid } & \text { GRS1980 }\end{array}$ & $\begin{array}{l}\text { Zone } \\
\text { Units }\end{array}$ & $\begin{array}{l}4976 \\
\text { FEET }\end{array}$ \\
\hline
\end{tabular}

\section{NARRATIVE FILE - COUNTY}

Abstract: County boundaries for the two counties in which RVAAP is located (Portage and Trumbull, Ohio).

Purpose: Cartographic. Part of data set delivered to Army Industrial Operations Command.

Limitations of data: Scale of 1:24,000 (Dave Wilburn, Ohio Environmental Protection Agency (OEPA), oral commun.).

Entity and attribute overview: COUNTY - name of county (10-digit character field).

Procedures used: Obtained digital data layer of Ohio townships from Dave Wilburn, OEPA. These townships were probably at a scale of 1:24,000, but Dave was not sure and thought that Public Utilities Commission of Ohio (PUCO) had been the agency that developed the data layer. USGS received it in state-plane south (zone 5001) coordinates and projected it into state-plane north (zone 4976) coordinates. The internal township boundaries were removed to form this data layer of the two counties.

Revisions: First.

Reviews applied to data: None. We assume that the OEPA and PUCO developers reviewed the data set.

Related spatial and tabular data sets: See the data layer TOWNSHIP.

References cited: None.

Notes: None.

Currentness reference: None.

Maintenance and update frequency: None planned.

Access constraints: None.

Data set credit: Public Utilities Commission of Ohio, Ohio Environmental Protection Agency, U.S. Army Industrial Operations Command.

Completeness report: Contains only 2 of Ohio's 88 counties.

Horizontal positional accuracy report: County boundary matches well with one of the edges of the plant boundary, which had been digitized from topographic quads at 1:24,000.

Vertical positional accuracy report: None.

Cloud cover: Not applicablc. 
Plant boundary - data layer BOUND

Description of DOUBLE precision data layer BOUND

\begin{tabular}{|c|c|c|}
\hline FEATURE CLASSES & $\begin{array}{l}\text { ARCS } \\
\text { POLYGONS } \\
\text { NODES }\end{array}$ & $\begin{array}{l}2 \\
2 \\
2\end{array}$ (TOPOLOGY) \\
\hline SECONDARY FEATURES & $\begin{array}{l}\text { Tics } \\
\text { Arc Segments } \\
\text { Polygon Labels }\end{array}$ & $\begin{array}{l}8 \\
255 \\
2\end{array}$ \\
\hline TOLERANCES & $\begin{array}{l}\text { Fuzzy }= \\
\text { Dangle = }\end{array}$ & $\begin{array}{l}132.710 \mathrm{~V} \\
0.000 \mathrm{~N}\end{array}$ \\
\hline \multicolumn{3}{|l|}{ DATA LAYER BOUNDARY } \\
\hline $\begin{array}{lc}X \min = & 2328260.789 \\
Y \min = & 548029.884\end{array}$ & $\begin{array}{l}X \max = \\
Y \max =\end{array}$ & $\begin{array}{c}2387831.824 \\
573288.352\end{array}$ \\
\hline \multicolumn{3}{|c|}{ COORDINATE SYSTEM DESCRIPTION } \\
\hline $\begin{array}{ll}\text { Projection } & \text { STATEPLANE } \\
\text { Datum } & \text { NAD83 } \\
\text { Spheroid } & \text { GRS1980 }\end{array}$ & $\begin{array}{l}\text { Zone } \\
\text { Units }\end{array}$ & $\begin{array}{l}4976 \\
\text { FEET }\end{array}$ \\
\hline
\end{tabular}

NARRATIVE FILE - BOUND

Abstract: Plant boundary, RVAAP, northeastern Ohio.

Keywords: Boundary.

Purpose: Visual purposes, perhaps some clipping of other data layers.

Limitations of data: Digitized at 1:24,000 scale. Not for property-assessment purposes.

Entity and attribute overview: None.

Procedures used: Digitized by USGS from 1:24,000 quadrangles: Ravenna (photoinspected 1977), Windham (photorevised 1979), and Newton Falls (photorevised 1979). Root-mean-square errors not recorded.

Revisions: 1. Before USGS review.

Reviews applied to data: 1. USGS, Columbus, Ohio, in-house, 12/5/96. Reviewer: Ralph Haefner. No problems.

Related spatial and tabular data sets: AREAAHJ delineates boundaries of some of the training areas.

Maintenance and update frequency: None expected.

Access constraints: None.

Data set credit: Ohio Army National Guard, Army Industrial Operations Command.

Completeness report: No omissions known.

Horizontal positional accuracy report: Should be satisfactory for illustration purposes but is not quantified.

Vertical positional accuracy report: Not applicable.

Cloud cover: Not applicable. 


\begin{tabular}{|c|c|c|}
\hline \multicolumn{3}{|c|}{$\begin{array}{l}\text { Municipal boundaries - data layer MUNICIP } \\
\text { Description of DOUBLE precision data layer MUNICIP }\end{array}$} \\
\hline FEATURE CLASSES & $\begin{array}{l}\text { ARCS } \\
\text { POLYGONS } \\
\text { NODES }\end{array}$ & $\begin{array}{l}3 \\
4 \\
3\end{array}$ (TOPOLOGY) \\
\hline SECONDARY FEATURES & $\begin{array}{l}\text { Tics } \\
\text { Arc Segments } \\
\text { Polygon Labels }\end{array}$ & $\begin{array}{l}8 \\
110 \\
3\end{array}$ \\
\hline TOLERANCES & $\begin{array}{l}\text { Fuzzy }= \\
\text { Dangle = }\end{array}$ & $\begin{array}{l}132.710 \mathrm{~V} \\
0.000 \mathrm{~N}\end{array}$ \\
\hline \multicolumn{3}{|l|}{ DATA LAYER BOUNDARY } \\
\hline $\begin{array}{lr}\mathrm{Xmin}= & 2312422.411 \\
\mathrm{Ymin}= & 541300.828\end{array}$ & $\begin{array}{l}X \max = \\
Y \max =\end{array}$ & $\begin{array}{c}2394532.811 \\
579529.772\end{array}$ \\
\hline \multicolumn{3}{|c|}{ COORDINATE SYSTEM DESCRIPTION } \\
\hline $\begin{array}{ll}\text { Projection } & \text { STATEPLANE } \\
\text { Datum } & \text { NAD83 } \\
\text { Spheroid } & \text { GRS1980 }\end{array}$ & $\begin{array}{l}\text { Zone } \\
\text { Units }\end{array}$ & $\begin{array}{l}4976 \\
\text { FEET }\end{array}$ \\
\hline
\end{tabular}

NARRATIVE FILE - MUNICIP

Abstract: City boundaries as depicted on the three quadrangle maps that span the RVAAP (Ravenna (photoinspected 1977), Windham (photorevised 1979), and Newton Falls (photorevised 1979), Ohio).

Kcywords: Municipality.

Purpose: Illustrative purposes only.

Limitations of data: Scale 1:24,000. State-plane projection.

Entity and attribute Overview: NAME - name of city.

Procedures used: Digitized by USGS from the quadrangle maps mentioned above. Digitizing precision not recorded.

Revisions: 1. Before USGS, Columbus, Ohio, in-house review.

Reviews applied to data: I. USGS in-house review, 12/5/96. Reviewer: Ralph Haefner. No problems.

Related spatial and tabular data sets: None.

References cited: None.

Notes: None.

Currentness reference: None.

Maintenance and update frequency: Not expected.

Access constraints: None.

Data set credit: Ohio Army National Guard and Army Industrial Operations Command.

Horizontal positional accuracy report: None.

Vertical positional accuracy report: None.

Cloud cover: Not applicable. 


\begin{tabular}{|c|c|c|}
\hline FEATURE CLASSES & $\begin{array}{l}\text { ARCS } \\
\text { POLYGONS } \\
\text { NODES } \\
\text { ANNOTATIONS }\end{array}$ & $\begin{array}{l}110 \\
43 \text { (TOPOLOGY) } \\
69 \\
1656\end{array}$ \\
\hline SECONDARY FEATURES & $\begin{array}{l}\text { Tics } \\
\text { Arc Segments } \\
\text { Polygon Labels }\end{array}$ & $\begin{array}{l}53 \\
559 \\
45\end{array}$ \\
\hline TOLERANCES & $\begin{array}{l}\text { Fuzzy }= \\
\text { Dangle = }\end{array}$ & $\begin{array}{l}328.155 \mathrm{~V} \\
328.155 \mathrm{~N}\end{array}$ \\
\hline $\begin{array}{lc}\text { DATA LAYER BOUNDARY } \\
\mathrm{Xmin}= & 2272948.500 \\
\mathrm{Ymin}= & 483601.781\end{array}$ & $\begin{array}{l}\mathrm{X} \max = \\
Y \max =\end{array}$ & $\begin{array}{l}2514171.250 \\
674502.000\end{array}$ \\
\hline $\begin{array}{ll}\text { COORDINATE SYSTEM DESCRI } \\
\text { Projection } & \text { STATEPLANE } \\
\text { Datum } & \text { NAD83 } \\
\text { Spheroid } & \text { GRS } 1980\end{array}$ & $\begin{array}{l}\text { ION } \\
\text { Zone } \\
\text { Units }\end{array}$ & $\begin{array}{l}4976 \\
\text { FEET }\end{array}$ \\
\hline
\end{tabular}

\section{NAARATIVE FILE - TOWNSHIP}

Abstract: Boundaries of townships of Portage and Trumbull Counties, Ohio.

Purpose: Cartographic. Forms part of a data set provided to U.S. Army Industrial Operations Command.

Limitations of data: Scale probably 1:24,000, according to Dave Wilburn, Ohio Environmental Protection Agency (OEPA).

Entity and attribute overview: DBCODE - unknown reference to an unknown table (USGS was not the originating agency for this data layer). TWN_NAME - name of township. COUNTY - name of county.

Procedures used: Obtained the township data layer in state-plane south coordinates from Dave Wilburn, OEPA. He thought Public Utilities Commission of Ohio (PUCO) was the originating agency. Map scale probably 1:24,000. Projected the data layer into state-plane north coordinates. This TOWNSHIP data layer is a subset of a statewide data layer of townships in Ohio's counties.

Revisions: None.

Reviews applied to data: None by USGS, on the assumption that the originating agency reviewed it.

Related spatial and tabular data sets: None.

References cited: None.

Notes: None.

Currentness reference: None.

Maintenance and update frequency: None planned.

Access constraints: None.

Data set credit: Ohio Environmental Protection Agency, Public Utilities Commission of Ohio, U.S. Army Industrial Operations Command.

Completeness report: None.

Horizontal positional accuracy report: None.

Vertical positional accuracy report: None.

Cloud cover: Not applicable. 
Roads - data layer ROADS

Description of DOUBLE precision data layer ROADS

\begin{tabular}{|c|c|c|}
\hline FEATURE CLASSES & $\begin{array}{l}\text { ARCS } \\
\text { NODES }\end{array}$ & $\begin{array}{l}1219 \text { (TOPOLOGY) } \\
991\end{array}$ \\
\hline SECONDARY FEATURES & $\begin{array}{l}\text { Tics } \\
\text { Arc Segments }\end{array}$ & $\begin{array}{l}43 \\
3980\end{array}$ \\
\hline TOLERANCES & $\begin{array}{l}\text { Fuzzy }= \\
\text { Dangle = }\end{array}$ & $\begin{array}{l}10.391 \mathrm{~N} \\
0.000 \mathrm{~N}\end{array}$ \\
\hline $\begin{array}{lc}\text { DATA LAYER BOUNDARY } \\
\mathrm{Xmin}= & 2312263.653 \\
\mathrm{Ymin}= & 533819.868\end{array}$ & $\begin{array}{l}\mathrm{X} \max = \\
\mathrm{Ymax}=\end{array}$ & $\begin{array}{l}2416177.659 \\
581037.643\end{array}$ \\
\hline $\begin{array}{ll}\text { COORDINATE SYSTEM DESCR } \\
\text { Projection } & \text { STATEPLANE } \\
\text { Datum } & \text { NAD83 } \\
\text { Spheroid } & \text { GRS } 1980\end{array}$ & $\begin{array}{l}\text { ION } \\
\text { Zone } \\
\text { Units }\end{array}$ & $\begin{array}{l}4976 \\
\text { FEET }\end{array}$ \\
\hline
\end{tabular}

NARRATIVE FILE - ROADS

Abstract: Data layer of roads in three quadrangles surrounding RVAAP in northeastern Ohio.

Keywords: Roads.

Purpose: Provide a base layer of information; illustrative.

Limitations of data: Digitized at 1:24,000. Root-mean-square errors associated with digitizing not recorded.

Entity and attribute overview: NAME - name of road, according to quadrangles.

Procedures used: Digitized by USGS from Ravenna (photoinspected 1977), Windham (photorevised 1979), and Newton Falls (photorevised 1979) 1:24,000-scale topographic quadrangle maps.

Revisions: 1. Before USGS review.

Reviews applied to data: 1. USGS, Columbus, Ohio, in-house, 12/5/96. Reviewer: Ralph Haefner. Comments: Not all roads outside the boundary were digitized. Selection was mostly arbitrary, except all major roads were digitized. Not all roads were named, but as many as could be, were.

Related spatial and tabular data sets: None.

References cited: None.

Notes: None.

Currentness reference: None.

Maintenance and update frequency: None expected.

Access constraints: None.

Data set credit (for funding): Ohio Army National Guard and Army Industrial Operations Command.

Completeness report: Small roads within city limits were excluded, but every road on RVAAP and the surrounding countryside was included.

Horizontal positional accuracy report: Satisfactory. No documentation for this.

Vertical positional accuracy report: Not applicable. 


\begin{tabular}{|c|c|c|}
\hline \multicolumn{3}{|c|}{$\begin{array}{l}\text { Railroads - data layer RAILROAD } \\
\text { Description of DOUBLE precision data layer RAILROAD }\end{array}$} \\
\hline FEATURE CLASSES & $\begin{array}{l}\text { ARCS } \\
\text { NODES }\end{array}$ & $\begin{array}{l}352 \text { (TOPOLOGY) } \\
298\end{array}$ \\
\hline SECONDARY FEATURES & $\begin{array}{l}\text { Tics } \\
\text { Arc Segments }\end{array}$ & $\begin{array}{l}8 \\
2023\end{array}$ \\
\hline TOLERANCES & $\begin{array}{l}\text { Fuzzy }= \\
\text { Dangle }=\end{array}$ & $\begin{array}{l}10.328 \mathrm{~N} \\
0.000 \mathrm{~N}\end{array}$ \\
\hline $\begin{array}{lc}\text { DATA LAYER BOUNDARY } \\
\mathrm{Xmin}= & 2312710.070 \\
\mathrm{Ymin}= & 533850.829\end{array}$ & $\begin{array}{l}X \max = \\
Y \max =\end{array}$ & $\begin{array}{l}2459861.212 \\
580595.215\end{array}$ \\
\hline $\begin{array}{ll}\text { COORDINATE SYSTEM DESCRII } \\
\text { Projection } & \text { STATEPLANE } \\
\text { Datum } & \text { NAD83 } \\
\text { Spheroid } & \text { GRS } 1980\end{array}$ & $\begin{array}{l}\text { ION } \\
\text { Zone } \\
\text { Units }\end{array}$ & $\begin{array}{l}4976 \\
\text { FEET }\end{array}$ \\
\hline
\end{tabular}

NARRATIVE FILE - RAILROAD

Abstract: This line data layer is to provide an illustrative data layer of railroads on and around RVAAP, northeastern Ohio.

Keywords: Railroad.

Purpose: Illustrative.

Limitations of data: Scale 1:24,000.

Entity and attribute overview: OWNER lists the name of the railroad company, according to the topographic quads.

Procedures used: Digitized by USGS from Ravenna (photoinspected 1977), Windham (photorevised 1979), and Newton Falls (photorevised 1979) 1:24,000-scale topographic quadrangle maps. Digitizing root-mean-square errors not recorded.

Revisions: I. Before USGS in-house review.

Reviews applied to data: I. USGS, Columbus, Ohio, in-house, 12/5/96. Reviewer: Ralph Haefner. Comments: Some short arcs were missing. These were added. Other linework was corrected.

Related spatial and tabular data sets: None.

References cited: None.

Currentness reference: None.

Maintenance and update frequency: None expected.

Access constraints: None.

Data set credit (for funding): Ohio Army National Guard and Army Industrial Operations Command.

Completeness report: None.

Horizontal positional accuracy report: None.

Vertical positional accuracy report: None.

Cloud cover: Not applicable. 
Off-plant pipelines - data layer PIPELINE

Description of DOUBLE precision data layer PIPELINE

\begin{tabular}{|c|c|c|}
\hline FEATURE CLASSES & $\begin{array}{l}\text { ARCS } \\
\text { NODES }\end{array}$ & $\begin{array}{l}14 \text { (TOPOLOGY) } \\
27\end{array}$ \\
\hline SECONDARY FEATURES & $\begin{array}{l}\text { Tics } \\
\text { Arc Segments }\end{array}$ & $\begin{array}{l}8 \\
300\end{array}$ \\
\hline TOLERANCES & $\begin{array}{l}\text { Fuzzy }= \\
\text { Dangle }=\end{array}$ & $\begin{array}{l}10.340 \mathrm{~N} \\
0.000 \mathrm{~N}\end{array}$ \\
\hline $\begin{array}{lc}\text { DATA LAYER BOUNDARY } \\
\mathrm{Xmin}= & 2312493.694 \\
Y \min = & 539310.039\end{array}$ & $\begin{array}{l}X \max = \\
Y \max =\end{array}$ & $\begin{array}{l}2415896.369 \\
580592.054\end{array}$ \\
\hline $\begin{array}{ll}\text { COORDINATE } & \text { SYSTEM DESCRI } \\
\text { Projection } & \text { STATEPLANE } \\
\text { Datum } & \text { NAD83 } \\
\text { Spheroid } & \text { GRS } 1980\end{array}$ & $\begin{array}{l}\text { ION } \\
\text { Zone } \\
\text { Units }\end{array}$ & $\begin{array}{l}4976 \\
\text { FEET }\end{array}$ \\
\hline
\end{tabular}

Narrative file - PIPELINE

Abstract: Data layer depicts pipelines as drawn on Ravenna (photoinspected 1977), Windham (photorevised 1979), and Newton Falls (photorevised 1979), Ohio, 1:24,000-scale topographic quadrangles.

Keywords: Pipe. None.

Purpose: Illustrative purposes only.

Limitations of data: Scale 1:24,000. Not intended for engineering or construction.

Entity and attribute overview:

Procedures used: Digitized by USGS from the quads mentioned above. Digitizing errors not recorded.

Revisions: 1. Before USGS in-house review.

Reviews applied to data: 1. USGS in-house, 12/5/96. Reviewer: Ralph Haefner. No problems, after the data layer was built as a line data layer.

Related spatial and tabular data sets: None.

References cited: None.

Notes: None.

Currentness reference: None.

Maintenance and update frequency: None planned.

Access constraints: None.

Data set credit (for funding): Ohio Army National Guard and Army Industrial Operations Command.

Completeness report: None.

Horizontal positional accuracy report: None.

Vertical positional accuracy report: None.

Cloud cover: Not applicable. 


\begin{tabular}{|c|c|c|}
\hline \multicolumn{3}{|c|}{$\begin{array}{l}\text { Surface drainage and lakes - data layer DRAINAGE } \\
\text { Description of DOUBLE precision data layer DRAINAGE }\end{array}$} \\
\hline \multicolumn{3}{|c|}{$\begin{array}{lll}\text { FEATURE CLASSES } & \text { ARCS } & 1268 \text { (TOPOLOGY) } \\
& \text { POLYGONS } & 619 \text { (TOPOLOGY) } \\
& \text { NODES } & 1156\end{array}$} \\
\hline SECONDARY FEATURES & $\begin{array}{l}\text { Tics } \\
\text { Arc Segments } \\
\text { Polygon Labels }\end{array}$ & $\begin{array}{l}8 \\
48552 \\
618\end{array}$ \\
\hline TOLERANCES & $\begin{array}{l}\text { Fuzzy }= \\
\text { Dangle }=\end{array}$ & $\begin{array}{l}132.710 \mathrm{~V} \\
0.000 \mathrm{~N}\end{array}$ \\
\hline $\begin{array}{ll}X \min = & 2312271.866 \\
Y \min = & 533938.730\end{array}$ & $\begin{array}{l}X \max = \\
Y \max =\end{array}$ & $\begin{array}{l}2416146.741 \\
581009.170\end{array}$ \\
\hline \multicolumn{3}{|c|}{$\begin{array}{lll}\text { COORDINATE SYSTEM DESCRIPTION } \\
\text { Projection } & \text { STATEPLANE } & \text { Zone } \\
\text { Datum } & \text { NAD83 } & \text { Units } \\
\text { Spheroid GRS } 1980 & & \end{array}$} \\
\hline
\end{tabular}

\section{NARRATIVE FILE - DRAINAGE}

Abstract: Surface-water features depicted on Ravenna, Windham, and Newton Falls, Ohio, quadrangles (dates bclow). Source maps 1:24,000 scale. Digitized in Albers meters projection, projected to stateplane coordinates.

Purpose: Part of digital spatial data base for Army Industrial Operations Command.

Limitations of data: Scale 1:24,000.

Entity and attribute overview:

Polygons NAME - name of polygon features, where applicable or known

TYPE - type of feature (lake, river, island)

SYM - drawing symbol. Water features $=4$, islands $=0$

Arcs

NAME - name of line feature, where known or applicable

TYPE - type of stream $(I=$ intermittent, all others = blank $)$

Procedures used: These featur es were digitized by USGS from Ravenna (photoinspected 1977), Windham (photorevised 1979), and Newton Falls (photorevised 1979) topographic quads, Ohio. Root-mean-square errors: Ravenna: 0.006 (3.64 m); Windham: $0.002(1.45 \mathrm{~m})$; Newton Falls: $0.002(1.98 \mathrm{~m})$. Digitizing was done with NODESNAP CLOSEST 10 and WEEDTOL 0.01 parameters. Edgematch effects were cleaned up in ArcEdit after all three quads were digitized. Attribute coding was done in ArcEdit after all three quads were digitized.

Revisions: This is the second drainage data layer produced for the Ravenna area by USGS. The first was done for a project between USGS and Ohio Army National Guard. It was a 1:100,000 U.S. Environnental Protection Agency RF3 file that had been modified a little. The data described herein should be more accurate (especially in linework) than those in the previous file because of smaller scale.

Reviews applied to data: USGS in-house, 9/25/97. Reviewers: Ralph Haefner and Dan Button. Comments: None.

Related spatial and tabular data sets: None.

References cited: None.

Notes: None.

Currentness reference: None.

Maintenance and update frequency: None planned.

Access constraints: None.

Data set credit: Funded by and for the use of Army Industrial Operations Command.

Completeness report: Some of the smaller islands and (or) ponds could have been omitted.

Horizontal positional accuracy report: None.

Vertical positional accuracy report: None. 


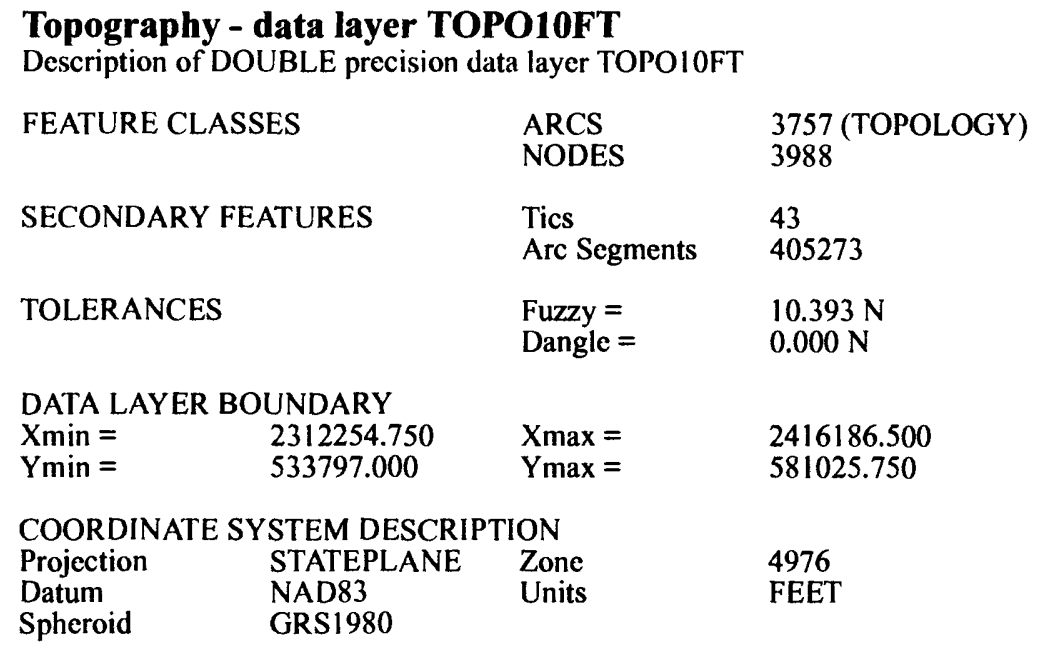

\section{NARRATIVE FILE - TOPO10FT}

Abstract: Land-surface altitude in quadrangles surrounding RVAAP, northeastern Ohio.

Purpose: 1. Cartographic. 2. Should be useful for construction and other projects.

Limitations of data: Scale 1:24,000. No documentation from originating agency.

Entity and attribute overview: ELEV - land-surface altitude, in feet above sea level.

Procedures used: Hypsography digital line graphs (DLG's) for Ravenna, Windham, and Newton Falls (dates unknown) quads were downloaded from Ohio Department of Administrative Services web site, ftp.geodata.state.oh.us. These data layers were in UTM meters. Each quad was converted from DLG to ARC/INFO format using the standard conversion option of DLGARC. Each was then projected to state-plane north coordinates, datum NAD83. ELEV was assigned to each line by relate to the *.ACODE file. The bounding box of each data layer was deleted, and the data layers were joined together in ARCEDIT using the GET command.

Revisions: None.

Reviews applied to data: An in-house review was done; assumed that the data set was checked by the originating agency.

Related spatial and tabular data sets: None.

References cited: None.

Notes: See http://www.geodata.state.oh.us/dlg/

Currentness reference: None.

Maintenance and update frequency: None planned.

Access constraints: None.

Data set credit: Ohio GIS Support Center (they may have created the data layer).

Completeness report: None.

Horizontal positional accuracy report: Topographic lines had been digitized for parts of RVAAP as part of a previous project. The contours in this downloaded data layer lined up well with those created previously.

Vertical positional accuracy report: None.

Cloud cover: None. 
Soils - data layer SOILS

Description of DOUBLE precision data layer SOILS

\begin{tabular}{|c|c|c|}
\hline FEATURE CLASSES & $\begin{array}{l}\text { ARCS } \\
\text { POLYGONS } \\
\text { NODES }\end{array}$ & $\begin{array}{l}3078 \\
1093 \text { (TOPOLOGY) } \\
2127\end{array}$ \\
\hline SECONDARY FEATURES & $\begin{array}{l}\text { Tics } \\
\text { Arc Segments } \\
\text { Polygon Labels }\end{array}$ & $\begin{array}{l}36 \\
75385 \\
1092\end{array}$ \\
\hline TOLERANCES & $\begin{array}{l}\text { Fuzzy }= \\
\text { Dangle }=\end{array}$ & $\begin{array}{l}123.602 \mathrm{~V} \\
0.000 \mathrm{~N}\end{array}$ \\
\hline DATA LAYER BOUNDARY & & \\
\hline $\begin{array}{ll}\mathrm{Xmin}= & 2328852.321 \\
\mathrm{Ymin}= & 542029.507\end{array}$ & $\begin{array}{l}X \max = \\
Y \max =\end{array}$ & $\begin{array}{l}2393309.641 \\
583054.290\end{array}$ \\
\hline $\begin{array}{ll}\text { COORDINATE } & \text { SYSTEM DESCRI } \\
\text { Projection } & \text { STATEPLANE } \\
\text { Datum } & \text { NAD83 } \\
\text { Spheroid }= & \text { GRS } 1980\end{array}$ & $\begin{array}{l}\text { ION } \\
\text { Zone } \\
\text { Units }\end{array}$ & $\begin{array}{l}4976 \\
\text { FEET }\end{array}$ \\
\hline
\end{tabular}

NARRATIVE FILE - SOILS

Abstract: Data layer of Soil Conservation Service (SCS) soil designations on RVAAP, Ohio.

Keywords: Soil type, hydric.

Purpose: One of the main criteria used to define wetlands is the presence of hydric soils. All the soils polygons were digitized so that this data layer could be used in overlays with other data layers and could be used for wetland mapping.

Limitations of data: This was digitized by USGS at 1:15,840. See digitizing caveats below.

Entity and attribute overview:

SSYM - SCS soil type code. Full descriptions can be found in the documents cited below.

PSYM - an arbitrary plotting symbol for shading.

HYDRIC - flag as to whether a soil type is hydric.

Procedures used: Digitizing-Loose-leaf maps were digitized in inches, with the intent of transforming them to real-world coordinates later. Tics were created at 10" intervals in the y-direction and 15" intervals in the x-direction (pretty nearly the dimensions of one of the map sheets). Digitizing root-mean-square (RMS) errors ranged from 0.003 (Trumbull County sheet 57) to 0.008 (Portage County sheet 30). Transforming - Empty data layers were created from ROADS data layer, and tics were added at points that were equally discernible on the Ravenna/Windham/Newton Falls quads and the aerial photographs over which the soils lines were drawn. At least 4 tics were added for each SCS sheet and corresponding locations in the empty data layers. The transformation parameters are presented below: (for example, "soil23" corresponds to Portage County sheet 23).

Transforming coordinates for data layer soil23

Scale $(X, Y)=(403.381,403.885)$ Skew $($ degrees $)=(0.017)$

Rotation (degrees) $=(3.091)$ Translation $=(229866.601,4312749.036)$

RMS Error (input,output) $=(0.002,0.732)$

Affine

$X=A x+B y+C$

$\mathrm{A}=$

$\mathrm{Y}=\mathrm{Dx}+\mathrm{Ey}+\mathrm{F}$

$A=$

402.794

$\mathrm{B}=$

229866.601

$\mathrm{D}=$

$-21.662$

$\mathrm{E}=$

403.303

$\mathrm{F}=$

21.753

tic id

input $x$

input $y$

$36-30.867$

output y

$\mathrm{x}$ error

$y$ error

28.463

241682.832

4324899.093

-------

37

44.893

27.861

247345.852

4324962.837

0.355

0.653

38

30.448

20.772

241681.142

$432 \mathrm{I} 789.272$

$-0.352$

$-0.647$

39

44.796

20.083

247474.759

4321822.466

$-0.347$

$-0.639$

0.344

0.633 


\section{Soils - data layer SOILS-Continued}

Transforming coordinates for data layer soil24

Scale $(X, Y)=(404.381,405.464)$ Skew $($ degrees $)=(-0.117)$

Rotation (degrees) $=(3.287)$ Translation $=(229770.313,4312652.964)$

RMS Error (input,output) $=(0.021,8.473)$

Affine

$X=A x+B y+C$
$Y=D x+F y+F$

$A=$
$C=$

$\mathrm{C}=\quad 229770.313$

$\mathrm{B}=$

$-24.069$

$\mathrm{E}=$

404.750

$\mathrm{D}=$
$\mathrm{F}=$

23.184

tic id

input $x$

input $y$

44

output $x$

output y

4312652.964

45

45.208

247322.094

$45 \quad 57.960$

252490.600

46

45.346

29.336

4325576.510

27.831

4325258.603

$-6.532$

$-1.876$

20.673

247573.141

4322069.659

9.386

2.696

47

57.898
252629.895

21.789

6.650

1.910

4322816.966

$-9.504$

$-2.730$

$==============$

Scale $(X, Y)=(402.341,404.032)$ Skew $($ degrees $)=(-0.137)$

Rotation $($ degrees $)=(3.061)$ Translation $=(230024.676,4312857.820)$

RMS Error (input,output) $=(0.010,4.158)$

Affine $X=A x+B y+C$
$Y=D x+E y+F$

$\mathrm{A}=$ 401.767

$\mathrm{B}=$

230024.676

$\mathrm{D}=$

$-22.541$

$C=$

403.404

$F=$

21.488

$\mathrm{E}=$

input $x$

input $y$

tic id

output $x$

output y

$x$ error

$y$ error

20

15.769

18.187

235949.097

21

29.329

4320529.708

0.971

3.852

18.904

241382.768

4321117.870

0.971

$-3.727$

22

236009.597

10.445

$-0.939$

$-4.322$

23

27.511

4317408.319

$-1.090$

4.198

Transforming coordinates for data layer soil29

Scale $(X, Y)=(401.417,402.158)$ Skew (degrees $)=(0.173)$

Rotation (degrees) $=(3.118)$ Translation $=(229922.236,4312882.157)$

RMS Error (input,output) $=(0.014,5.776)$

Affine

$\mathrm{A}=$
$\mathrm{C}=$

$X=A x+B y+C$

$\mathrm{Y}=\mathrm{Dx}+\mathrm{Ey}+\mathrm{F}$

$\mathrm{E}=$

400.823

229922.236

$B=$

$\mathrm{D}=$

$-20.661$

401.628

$\mathrm{F}=$

21.835

tic id

input $x$

\begin{tabular}{ll} 
& output $x$ \\
\hline 32 & 30.796 \\
\hline
\end{tabular}

input $y$

output y

$y$ error

17.902

241892.604

4320739.641

3.361

4.846

33

44.522

19.452

4321670.761

$-2.825$

$-4.073$

34

68.437

10.380

4317755.869

$-3.727$

$-5.373$

35

242550.745

44.395

10.488

247496.723

4318059.136

3.190

4.600 
Soils - data layer SOILS - Continued

Transforming coordinates for data layer soil 30

Scale $(X, Y)=(404.350,403.756)$ Skew $($ degrees $)=(0.325)$

Rotation (degrees) $=(3.174)$ Translation $=(229711.033,4312798.692)$

RMS Error (input,output) $=(0.046,18.458)$

$\begin{array}{ll}\text { Affine } & X=A x+B y+C \\ Y=D x+E y+F\end{array}$

$A=$

$\mathrm{C}=$

403.729

229711.033

$\mathrm{E}=$

403.264

$\begin{array}{lr}B= & -20.069 \\ D= & 22.389 \\ F= & 4312798.692^{2}\end{array}$

tic id

input $x \quad$ input $y$

40

output $x$

output y

$x$ error

y error

$47.308 \quad 17.692$

41

248451.453

59.431

253341.110

42

45.388

247815.953

43

59.159
253374.475

4320972.074

4.180

20.436

18.316

4321533.637

$-3.694$

$-18.060$

11.127

4318320.035

$-3.670$

$-17.943$

10.849

4318482.772

3.184

15.567

\begin{tabular}{|c|c|c|c|c|}
\hline \multicolumn{5}{|c|}{$\begin{array}{l}\text { Transforming coordinates for data layer soil34 } \\
\text { Scale }(X, Y)=(403.614,400.952) \text { Skew }(\text { degrees })=(0.300) \\
\text { Rotation }(\text { degrees })=(3.132) \text { Translation }=(229971.627,4313000.666) \\
\text { RMS Error (input,output) }=(0.023,9.428)\end{array}$} \\
\hline Affine & \multicolumn{4}{|c|}{$\begin{array}{l}X=A x+B y+C \\
Y=D x+E y+F\end{array}$} \\
\hline $\begin{array}{l}A= \\
C= \\
E=\end{array}$ & $\begin{array}{l}403.011 \\
229971.627 \\
400.468\end{array}$ & $\begin{array}{l}B= \\
D= \\
F=\end{array}$ & $\begin{array}{l}-19.813 \\
22.054 \\
431300\end{array}$ & \\
\hline tic id & $\begin{array}{l}\text { input } x \\
\text { output } x\end{array}$ & $\begin{array}{l}\text { input y } \\
\text { output } y\end{array}$ & $\mathrm{x}$ error & y crror \\
\hline 24 & 15.196 & 9.303 & & \\
\hline & $\begin{array}{l}235917.712 \\
29743\end{array}$ & $\begin{array}{l}4317058.917 \\
8.947\end{array}$ & -6.207 & 2.256 \\
\hline 25 & 241770.739 & 4317243.416 & 10.462 & -3.802 \\
\hline \multirow[t]{2}{*}{26} & 16.124 & 1.529 & & \\
\hline & 236432.680 & 4313970.942 & 6.735 & -2.44 \\
\hline 27 & $\begin{array}{l}29.613 \\
241833652\end{array}$ & $\begin{array}{l}4.200 \\
431533 \mid 795\end{array}$ & -10990 & 3994 \\
\hline
\end{tabular}

Transforming coordinates for data layer soil35

Scale $(X, Y)=(402.421,401.660)$ Skew $($ degrees $)=(0.197)$

Rotation $($ degrees $)=(3.109)$ Translation $=(229896.530,4312991.476)$

RMS Error (input,output) $=(0.005,1.808)$

Affine

$A=$

$\mathrm{C}=$

$\mathrm{E}=$

tic id

28

29

30

31

$\begin{array}{ll} & 43.858 \\ & 247465.727\end{array}$

401.829

229896.530

input $x$

output $x$

30.929

242137.510

44.123

247443.409

31.055
$X=A x+B y+C$

$\mathrm{Y}=\mathrm{Dx}+\mathrm{Ey}+\mathrm{F}$

B =

$\mathrm{D}=$

$\mathrm{F}=$

$-20.409$

21.827

4312991.476

input $y$

input y

9.201

4317356.013

8.919

4317534.129

0.213

4313756.248

2.702

4315030.629 $x$ error

0.554

0.810

0.576

$-0.832$ y error

1.309

$-1.913$

$-1.361$

1.965 


\section{Soils - data layer SOILS - Continued}

Transforming coordinates for data layer tsoil ln

Scale $(X, Y)=(400.104,399.922)$ Skew $($ degrees $)=(-0.192)$

Rotation (degrees) $=(1.547)$ Translation $=(251149.229,4318769.210)$

RMS Error (input,output) $=(0.003,1.346)$

Affine

$X=A x+B y+C$
$Y=D x+E y+F$

$A=$

399.958

$\mathrm{C}=$
$\mathrm{E}=$

251149.229

$\mathrm{B}=$

$-12.136$

$\mathrm{E}=$

399.740

$\mathrm{D}=$
$\mathrm{F}=$

10.802

tic id

input $x$

output $x$

input $y$

output $y \quad x$ error

1067

3.736

252448.830

16.028

4325215.805

$x$ error

-........-.

13.229

10.919

255355.985

1068

8.067

4324176.422

0.104

0.599

9.952

254254.393

47

4.005

4322832.614

$-0.188$

$-1.083$

10.014

252629.895

4322816.966

0.327

1.884

$-0.243$

$-1.399$

Transforming coordinates for data layer tsoil2s

Scale $(X, Y)=(401.333,403.338)$ Skew $($ degrees $)=(-0.221)$

Rotation $($ degrees $)=(1.871)$ Translation $=(251140.166,4318864.620)$

RMS Error (input,output) $=(0.016,6.454)$

Aftine

$\mathrm{X}=\mathrm{Ax}+\mathrm{By}+\mathrm{C}$

$\mathrm{A}=$

$\mathrm{Y}=\mathrm{Dx}+\mathrm{Ey}+\mathrm{F}$

$\mathrm{C}=$

401.119

$\mathrm{B}=$

$-14.719$

251140.166

$\mathrm{D}=$

13.102

$\mathrm{E}=$

403.073

$\mathrm{F}=$

4318864.620

tic id

input $x$

input $y$

output $x \quad$ output $y$

47

4.069

9.687

4322816.966

$x$ error

$y$ error

107

252629.895

7.480

255661.000

4322034.984

$-0.351$

5.505

6.413

253341.110

4321533.637

$-2.125$

$-4.367$

41

$\begin{array}{ll}253341.110 & 4321533 \\ 2.059 & 5.716\end{array}$

1070

251881.483

4321194.901

1.108

$-8.935$

3.788

254895.103

4320506.588

0.362

0.529

1.158

4319407.523

3.405

9.578

253384.808

$-2.399$

$-2.311$

The transformation of soil30 did not line up well with the others, but that was not surprising because it had such a large rootmean-square error. To get good line-matching at the boundaries, all of soil 30 was moved up (north) $30 \mathrm{~m}$ from where it had been transformed. Cleaning up all layers - Most of the line matches were satisfactory, and the edges were cleaned up using splitting of arcs and moving of nodes in ArcEdit. There were no glaring, hugely mismatched lines. Attributing-Once all the linework looked good, the data layer was built as polygons and labels were added. Attribute fields were added, and polygons were tagged with SSYM.

Revisions I. Before USGS review.

Reviews applied to data: 1. USGS, Columbus, Ohio, in-house, 12/5/96. Reviewer: Ralph Haefner. Comments: Attribute coding is laborious. True, but did not change.

Related spatial and tabular data sets: None. Those that were used to create this data layer were deleted.

Notes: Resultant data layer SOILS in state-plane feet.

References cited: Williams, N. L., 1992, Soil survey of Trumbull County, Ohio: U.S. Department of Agriculture, Soil Conservation Service, 256 p. with maps. Ritchie, A., Bauder, J.R., and Christman, R.L., 1978, Soil survey of Portage County, Ohio: U.S. Department of Agriculture, Soil Conservation Service, 116 p. with maps.

Access constraints: None.

Data set credit (for funding): Ohio Army National Guard and Army lndustrial Operations Command. 
Plant communities - data layer PLNTCM3

Description of DOUBLE precision data layer PLNTCM3

\begin{tabular}{|c|c|c|}
\hline FEATURE CLASSES & $\begin{array}{l}\text { ARCS } \\
\text { POLYGONS } \\
\text { NODES }\end{array}$ & $\begin{array}{l}2218 \\
786 \text { (TOPOLOGY) } \\
1459\end{array}$ \\
\hline SECONDARY FEATURES & $\begin{array}{l}\text { Tics } \\
\text { Arc Segments } \\
\text { Polygon Labels }\end{array}$ & $\begin{array}{l}6 \\
29656 \\
785\end{array}$ \\
\hline TOLERANCES & $\begin{array}{l}\text { Fuzzy }= \\
\text { Dangle }=\end{array}$ & $\begin{array}{l}3.814 \mathrm{~V} \\
0.000 \mathrm{~N}\end{array}$ \\
\hline $\begin{array}{lc}\text { DATA LAYER BOUNDARY } \\
\mathrm{Xmin}= & 2328260.789 \\
\mathrm{Ymin}= & 548029.884\end{array}$ & $\begin{array}{l}\mathrm{X} \max = \\
\mathrm{Ymax}=\end{array}$ & $\begin{array}{l}2387831.824 \\
573288.352\end{array}$ \\
\hline $\begin{array}{ll}\text { COORDINATE SYSTEM DESCRI } \\
\text { Projection } & \text { STATEPLANE } \\
\text { Datum } & \text { NAD83 } \\
\text { Spheroid } & \text { GRS } 1980\end{array}$ & $\begin{array}{l}\text { ION } \\
\text { Zone } \\
\text { Units }\end{array}$ & $\begin{array}{l}4976 \\
\text { FEET }\end{array}$ \\
\hline
\end{tabular}

NARRATIVE FILE - PLNTCM3

Abstract: Polygons (areas) designating dominant plant communities on RVAAP, northeastern Ohio.

Keywords: Plant species, wetlands.

Purpose: Several of the plant communities are indicative of the presence of wetlands. It was intended that this map should serve as a data layer that provides criteria for field examination of wetland areas.

Limitations of data: The source map scale was about $1: 23,256$. It was a blue-linc copy of the original field map, which is on filc at the Division of Natural Areas and Preserves, Ohio Department of Natural Resources (ODNR), under the management of Dan Rice. The source map was of poor quality. Some of the symbols and lines were difficult to read. The base map was a photoreduced version of a 1:12,000-scale general area map of RVAAP, and had roads, railroads, buildings, and streams on it. During review, the original document at ODNR was consulted for details, especially on attribute coding.

Entity and attribute overview:

COMSYM - plant-community symbol described by Andreas (1993) (see citation below).

SYMBOL - Integer version of COMSYM

$\begin{array}{lll}\text { COMSYM } & \text { SYMBOL } & \begin{array}{l}\text { DESCRIPTION } \\ \text { Submergent marsh } \\ 1\end{array} \\ 2 & 2 & \text { Floating-leaved marsh } \\ 3 & 3 & \text { Mixed emergent marsh } \\ 3 & 4 & \text { Cattail marsh } \\ 4 & 5 & \text { Sedge-grass meadow } \\ 5 & 6 & \text { Mixed shrub swamp } \\ 6 & 7 & \text { Buttonbush shrub swamp } \\ 7 & 8 & \text { Oak-maple swamp forest } \\ 8 & 9 & \text { Mixed swamp forest } \\ 9 & 10 & \text { Mixed flood-plain forest } \\ 10 & 11 & \text { Beech-sugar maple forest } \\ 11 & 12 & \text { Hemlock-white pine-hardwood forest } \\ 12 & 13 & \text { Oak-maple-tulip tree forest } \\ 13 & 14 & \text { Oak-hickory forest } \\ 14 & 15 & \text { Wet fields } \\ 15 & 16 & \text { Dry upland fields } \\ 16 & 16 & \text { Dry upland fields, transitional } \\ 16.1 & 17 & \text { Red maple woods } \\ 17 & 18 & \text { White ash-wild black cherry-red maple woods } \\ 18 & & \end{array}$

Procedures used: Digitized by USGS. Ticks were created in the corners of the source map. The lines were digitized; rootmean-square errors 0.004 and 0.003 . A data layer was prepared for TRANSFORM from the ROADS data layer. Because the source map had roads on it, we could define road-intersection points on the PLNTCOMM data layer and in the data layer to which the transformation would occur. Then we TRANSFORMed the data layer and added attribute data such as COMSYM and SYMBOL.

Revisions: I. Before USGS in-house review, when areal extent included training areas only. 2. After data layer was expanded to entire facility.

Reviews applied to data: I. USGS in-house review, 12/6/1996. Reviewer: Ralph Haefner. Comment: Linework good. Some polygons that are coded " 0.0 " are blank on the source map, whereas others are "clear cut." These were 


\section{Plant communities - data layer PLNTCM3 - Continued}

not distinguished in the data layer, because we did not want to add any additional plant-community codes to those Andreas (1993) defined.

2. After addition of the rest of RVAAP. Date: Aug 13, 1997. Reviewer: John Tertuliani. Scope of review: Comparison of digitized data layer and original field map on file at ODNR Division of Natural Areas and Preserves. Several community symbols were updated as a result of this review.

Related spatial and tabular data sets: PLNTCM3.LUT - a look-up table that describes the plant communities in PLNTCM3. The relate items are PLNTCM3.PAT:SYMBOL and PLNTCM3.LUT:SYM.

References cited: Andreas, B.K., 1993, Plant communities, in Species and Plant Communities Inventory, Ravenna Army Ammunition Plant. Report for Ravenna Army Ammunition Plant.

Currentness reference: Plant communities were described in 1992.

Maintenance and update frequency: None planned.

Access constraints: None.

Data set credit: Ohio Army National Guard and Army Industrial Operations Command and Bruce Daasch, manager.

Completeness report: Covers entire Plant.

Horizontal positional accuracy report: Poor in places, due to the digitizing errors, source map inadequacies, photo-reducing of the original base map, and TRANSFORM errors. Acceptable along the centerline (E-W) of the data layer.

Vertical positional accuracy report: Not applicable.

Cloud cover: Not applicable. 


\begin{tabular}{|c|c|c|}
\hline \multicolumn{3}{|c|}{$\begin{array}{l}\text { Wooded areas - data layer WOODLAND } \\
\text { Description of DOUBLE precision data layer WOODLAND }\end{array}$} \\
\hline FEATURE CLASSES & $\begin{array}{l}\text { ARCS } \\
\text { POLYGONS } \\
\text { NODES }\end{array}$ & $\begin{array}{l}455 \\
446 \text { (TOPOLOGY) } \\
455\end{array}$ \\
\hline SECONDARY FEATURES & $\begin{array}{l}\text { Tics } \\
\text { Arc Segments } \\
\text { Polygon Labels }\end{array}$ & $\begin{array}{l}8 \\
14968 \\
445\end{array}$ \\
\hline TOLERANCES & $\begin{array}{l}\text { Fuzzy }= \\
\text { Dangle }=\end{array}$ & $\begin{array}{l}132.710 \mathrm{~V} \\
0.000 \mathrm{~N}\end{array}$ \\
\hline \multicolumn{3}{|l|}{ DATA LAYER BOUNDARY } \\
\hline $\begin{array}{ll}\mathrm{Xmin}= & 2312670.617 \\
\mathrm{Ymin}= & 533797.235\end{array}$ & $\begin{array}{l}\mathrm{X} \max = \\
Y \max =\end{array}$ & $\begin{array}{l}2389156.450 \\
574744.751\end{array}$ \\
\hline \multicolumn{3}{|c|}{ COORDINATE SYSTEM DESCRIPTION } \\
\hline $\begin{array}{ll}\text { Projection } & \text { STATEPLANE } \\
\text { Datum } & \text { NAD83 } \\
\text { Spheroid } & \text { GRS1980 }\end{array}$ & $\begin{array}{l}\text { Zone } \\
\text { Units }\end{array}$ & $\begin{array}{l}4976 \\
\text { FEET }\end{array}$ \\
\hline
\end{tabular}

NARRATIVE FILE - WOODLAND

Abstract: Polygon data layer of wooded areas on RVAAP. The wooded areas were digitized from Ravenna, Windham, and Newton Falls, Ohio quads (see dates below), and the extent includes only RVAAP grounds.

Keywords: Woodland

Purpose: Illustrative mostly. Can be used as land-usc information.

Limitations of data: Scale 1:24,000. May be affected by further revisions to the topographic quads. Dates of the quads used in this effort werc: Ravenna, photoinspected 1977; Windham, photorevised 1979; Newton Falls, photorevised 1979.

Procedures used: Digitized by USGS from USGS topographic quads. Digitizing root-mean-square crrors not recorded.

Revisions: I. Before USGS in-house review.

Reviews applicd to data: 1. USGS, Columbus, Ohio, in-house review, 12/5/96. Revicwer: Ralph Haefner. Comments: Arcas described as "orchards" were not included in the digitizing. Some minor linework errors were corrected.

Related spatial and tabular data sets: None.

References cited: None.

Notes: None.

Currentness reference: None.

Maintenance and update frequency: None expected. Could be updated from photogrammetric methods.

Access constraints: None.

Data set credit: Ohio Army National Guard.

Completeness report: Digitized only for the areas on RVAAP. 
National Wetlands Inventory areas - data layer NWI2

Description of DOUBLE precision data layer NWI2

\begin{tabular}{|c|c|c|}
\hline FEATURE CLASSES & $\begin{array}{l}\text { ARCS } \\
\text { POLYGONS } \\
\text { NODES }\end{array}$ & $\begin{array}{l}381 \\
312 \text { (TOPOLOGY) } \\
354\end{array}$ \\
\hline SECONDARY FEATURES & $\begin{array}{l}\text { Tics } \\
\text { Arc Segments } \\
\text { Polygon Labcls }\end{array}$ & $\begin{array}{l}43 \\
13,673 \\
311\end{array}$ \\
\hline TOLERANCES & $\begin{array}{l}\text { Fuzzy = } \\
\text { Dangle = }\end{array}$ & $\begin{array}{l}123.602 \mathrm{~V} \\
0.000 \mathrm{~N}\end{array}$ \\
\hline $\begin{array}{lc}\text { DATA LAYER BOUNDARY } \\
\mathrm{Xmin}= & 2326122.614 \\
\mathrm{Ymin}= & 543182.444\end{array}$ & $\begin{array}{l}X \max = \\
Y \max =\end{array}$ & $\begin{array}{l}2389639.564 \\
574673.896\end{array}$ \\
\hline 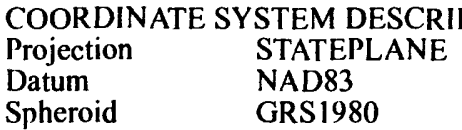 & $\begin{array}{l}\text { ON } \\
\text { Zone } \\
\text { Units }\end{array}$ & $\begin{array}{l}4976 \\
\text { FEET }\end{array}$ \\
\hline
\end{tabular}

NARRATIVE FILE - NWI2

Abstract: These data were digitized from National Wetlands Inventory (NWI) maps (Ravenna, Windham, and Newton Falls, Ohio, quadrangles) of RVAAP. Polygons and line features have attributes. The data were compiled digitally for the Ohio Army National Guard and Army Industrial Operations Command.

Keywords: Wetlands, GIS.

Purpose: This and several other data layers were used by USGS to make assessments of wetland areas on RVAAP.

Limitations of data: The data were digitized by USGS at a scale of $1: 24,000$.

Entity and attribute overview (specific attribute codes are described in the on-line documentation for this data layer):

SYS - Ecological system, described on the NWI maps.

SUBSYS - Ecological subsystem, described on the NWI maps.

CLASS - Class, described on the NWI maps.

SUBCLASS - Subclass, described on the NWI maps.

MODIFIER - Modifying term, described on the NWI maps.

CODE 1 - Primary composite wetland code designation.

CODE2 - Secondary composite wetland code designation.

Procedures used: The TICS for this data layer were based on corners of 1:24,000-scale USGS topographic quadrangle maps.

Root-mean-square digitizing errors for the three quadrangles ranged from $0.001-0.005$ in., correlating to errors of $0.9-2.8 \mathrm{~m}$. Polygon labels and line features were assigned attributes.

Revisions: 1. Before USGS review.

Reviews applied to data: 1. USGS review in Columbus, Ohio, 12/5/96. Reviewer: Ralph Haefner. Comments: INFO data structure could have been made easier with REDFINED items, but left as is. 2 . After data layer was expanded to all of plant, it was checked by Charles Schalk. A few small polygons that had been missing were added.

Related spatial and tabular data sets: None.

Notes: Units are state-plane feet.

Currentness reference: Aerial photographs and other mapping information were referenced as "1977" on the NWI quads.

References cited: U.S. Fish and Wildlife Service maps, 1977a, 1977b, 1977c.

Access constraints: None.

Data set credit: Ohio Army National Guard and Army Industrial Operations Command.

Completeness report: Covers entire plant.

Horizontal positional accuracy report: Ravenna quad-root-mean-square error $=0.005,2.787 \mathrm{~m}$. Windham quad-root-meansquare error $=0.001,0.875 \mathrm{~m}$. Newton Falls quad- root-mean-square error $=0.003,1.763 \mathrm{~m}$.

Vertical positional accuracy report: Not applicable.

Cloud cover: Not applicable. 


\begin{tabular}{|c|c|c|}
\hline \multicolumn{3}{|c|}{$\begin{array}{l}\text { Buildings - data layer BUILDING } \\
\text { Description of DOUBLE precision data layer BUILDING }\end{array}$} \\
\hline FEATURE CLASSES & $\begin{array}{l}\text { ARCS } \\
\text { POLYGONS } \\
\text { NODES }\end{array}$ & $\begin{array}{l}473 \\
467 \text { (Topology) } \\
472\end{array}$ \\
\hline SECONDARY FEATURES & $\begin{array}{l}\text { Tics } \\
\text { Arc Segments } \\
\text { Polygon Labels }\end{array}$ & $\begin{array}{l}41 \\
4615 \\
466\end{array}$ \\
\hline TOLERANCES & $\begin{array}{l}\text { Fuzzy }= \\
\text { Dangle = }\end{array}$ & $\begin{array}{l}4.944 \mathrm{~V} \\
0.000 \mathrm{~N}\end{array}$ \\
\hline $\begin{array}{lc}\text { DATA LAYER BOUNDARY } \\
\mathrm{Xmin}= & 2336958.281 \\
\mathrm{Ymin}= & 548300.108\end{array}$ & $\begin{array}{l}\mathrm{X} \max = \\
Y \max =\end{array}$ & $\begin{array}{l}2386399.771 \\
569921.172\end{array}$ \\
\hline $\begin{array}{ll}\text { COORDINATE SYSTEM DESCRI } \\
\text { Projection } & \text { STATEPLANE } \\
\text { Datum } & \text { NAD83 } \\
\text { Spheroid } & \text { GRS } 1980\end{array}$ & $\begin{array}{l}\text { ION } \\
\text { Zone } \\
\text { Units }\end{array}$ & $\begin{array}{l}4976 \\
\text { FEET }\end{array}$ \\
\hline
\end{tabular}

Narrative file - BUILDING

Abstract: Digitized boundaries of buildings on RVAAP, Ohio.

Purpose: To draw approximate building boundaries to be used as a base layer of information.

Limitations of data: Digitized from digital images of photos from the National High-Altitude Program (NHAP) that had been rectified to state-plane coordinates. Root-mean-square digitizing errors were not recorded. Many buildings at RVAAP are scheduled to be removed in the future (sometime after 1997). so as buildings are removed, this data layer will need to be revised.

Entity and attribute overview: None.

Procedures used: Black-and-white photographs from the USGS NHAP program were obtained for the Cleveland South area; these images included 7930-40 (4/14/94), 7934-214 (4/20/94), and 7930-82 (4/14/94). These photos were scanned at a resolution of $10 \mu \mathrm{m}$ and saved in the tag image file format (TIFF). For each of the images, a rectangular boundary was chosen and a polygon data layers was created; these data layer along with the ARC command RECTIFY were used to trim down the images. The images were then converted to a grid using the IMAGEGRID command so that they could be resampled to a more manageable size (in bytes). During the resampling process it was found that a cell size of 5 produced a good quality image at a reasonable storage size. The images were then converted back to the TIFF format using GRIDIMAGE and then converted to state plane coordinates using the REGISTER command. To make the images seamless when overlapped, the western- and easternmost images were clipped to rectangles. These images were then used as a background and the buildings were digitized into a polygon data layer from a monitor.

Revisions: None.

Reviews applied to data: USGS in-house, 7/97. Reviewer: Charles Schalk. Comments: Some of the building polygons had to be closed. Others had to be made more rectilinear. These corrections were done.

Related spatial and tabular data sets: None.

References cited: None.

Notes: None.

Currentness reference: Current as of 1994 , which is when aerial photographs were shot.

Maintenance and update frequency: None expected, but may be desirable as buildings are added or removed.

Access constraints: None.

Data set credit: U.S. Army Industrial Operations Command.

Completeness report: None.

Horizontal positional accuracy report: Within a few feet. The buildings data layers should be satisfactory.

Vertical positional accuracy report: Not applicable.

Cloud cover: None. 


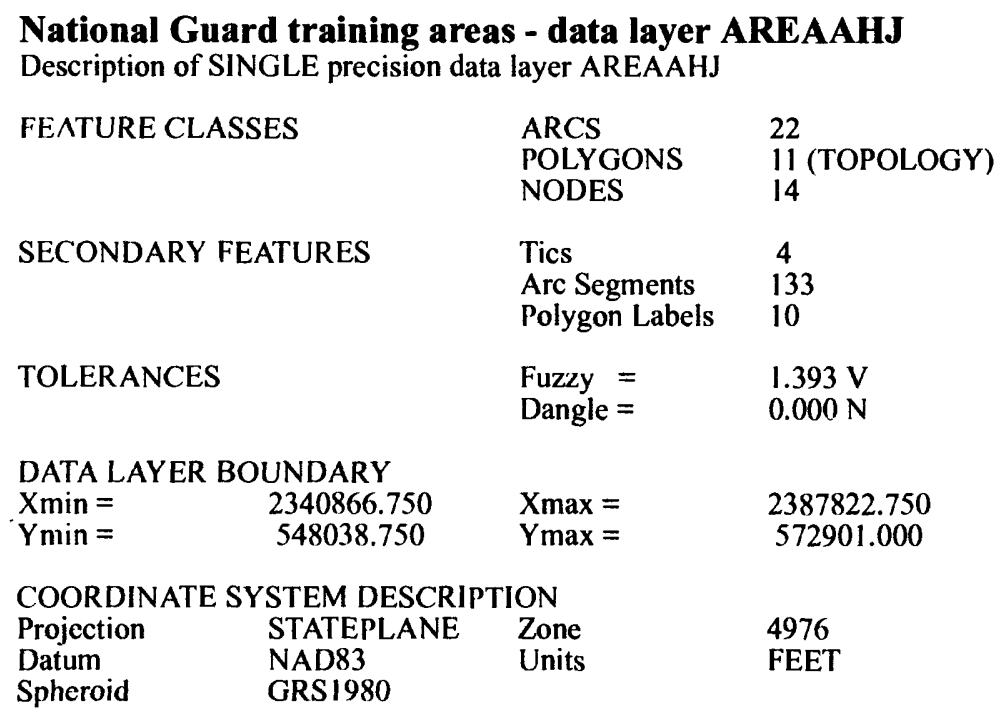

\section{NARRATIVE FILE - AREAAHJ}

Abstract: These data represent administrative areas A-H and J on RVAAP, Portage County, Ohio. Areas A-H and J are operated by Ohio Army National Guard (OHARNG) as of 10/1/96, whereas the rest of RVAAP (see BOUND) is operated by U.S. Army National Guard.

Keywords: Training area.

Purpose: Show the boundaries of areas operated by OHARNG. These areas will be used to clip other data layers to the specific limits needed by OHARNG.

Limitations of data: Scale 1:24,000. Valid as of 10/1/96.

Entity and attribute overview: NAME describes the administrative area. The polygon called "CO" is proposed for use by OHARNG.

Procedures used: Roads and other features were used by USGS to draw these boundaries. The boundary of Area $\mathrm{H}$ is most likely to be erroneous, but should be close, based on what we have seen on OHARNG maps.

Revisions: 1. Before USGS review. Lines were changed somewhat after a meeting with OHARNG, where we discovered houndaries were not inclusive enough. Boundaries were drawn to correspond with the boundary in the BOUND data layer, which was digitized from the Ravenna (photoinspected 1977), Windham (photorevised 1979), and Newton Falls (photorevised 1979) $1: 24,000$-scale topographic quadrangles.

Reviews applied to data: I. USGS, Columbus, Ohio, in-house, I2/5/96. Reviewer: Ralph Haefner. No problems.

Related spatial and tabular data sets: BOUND describes the entire RVAAP boundary.

Maintenance and update frequency: None expected.

Access constraints: None.

Data set credit: Ohio Army National Guard.

Completeness report: No omissions known.

Horizontal positional accuracy report: Should be satisfactory, but is not quantified.

Cloud cover: Not applicable. 